\title{
Campesinos andinos y políticas agrarias durante la Junta de Adelanto de Arica (Azapa, Lluta y la precordillera, 1959-1976)
}

\author{
Andean peasants and agrarian policies during the Advancement Council \\ (Junta de Adelanto) of Arica (Azapa, Lluta and foothills, 1959-1976)
}

Diego Quiroz Thompson ${ }^{1}$, Alberto Díaz Araya ${ }^{2}$, Luis Galdames Rosas ${ }^{3}$, Rodrigo Ruz Zagal ${ }^{4}$

\section{RESUMEN}

El presente artículo pretende desde una perspectiva histórica evidenciar por medio del análisis de los programas agropecuarios implementados por un organismo gubernamental de nivel local denominado Junta de Adelanto la participación e interacción que algunos segmentos indígenas del Departamento de Arica tuvieron con la estructura estatal y mercado regional entre 1959-1976. Para esto se procedió a la revisión de las políticas agrarias impulsadas por dicha institución.

Palabras clave: Campesinos andinos, políticas agrarias, Junta de Adelanto de Arica.

\section{ABSTRACT}

The present article tries from a historical perspective to demonstrate by means of the analysis of the agricultural programs implemented by a governmental organism of local level named Meeting of Advancement, the participation and interaction that some indigenous segments of Arica's Department, they had with the State structure and regional market between 1959-1976. For this one proceeded to the review of the agrarian policies stimulated by the above mentioned institution.

Key words: Andean peasants, agrarian policies, Junta de Adelanto de Arica.

\section{Introducción}

Durante el siglo XX, los campesinos e indígenas andinos del Departamento de Arica, que residían por centurias en ecologías diferenciadas como son las cabeceras de los valles de Azapa o Lluta, sierra de Huaylillas (o precordillera) y el altiplano, experimentaron una serie de procesos históricos, sociales, económicos y/o culturales, que identificamos como la "chilenización", la explotación minera, urbanización de las zonas costeras, intensificación de migraciones a la ciudad y desarrollo industrial de los centros urbanos ${ }^{1}$. Bajo estas premisas, este artículo examina la coyuntura socioeconómica que tiene lugar en el Departamento de Arica entre los años 1959 y 1976, para evidenciar lo que -a nuestro juicio- sería un proceso regional de intensificación de la participación de campesinos e indígenas andinos en las políticas agrarias del Estado chileno. La coyuntura a nivel local a la que hacemos alusión tiene que ver con la activación económica de la ciudad de Arica derivada de una política de excepción promovida durante el gobierno de Carlos Ibáñez del $\mathrm{Campo}^{2}$, que permitió -entre otras cosas- dotar de mayores recursos económicos al Departamento. Éstos excedentes fueron invertidos por una entidad denominada Junta de Adelanto de Arica (JAA), en un sinnúmero de obras que permitieron mejorar las condiciones de vida de la población. Puntualizando, la participación de los segmentos indígenas andinos en los programas agropecuarios implementados por la JAA en los valles de Lluta, Azapa y en la precordillera, y el contexto histórico en el que se insertan, constituyen los ejes analíticos que explora el artículo.

\section{La Junta de Adelanto de Arica y el desarrollo rural (1959-1976)}

En 1958 se creó la JAA, con personalidad jurídica de derecho público cuyo fin era "fomentar la producción y, en general, el progreso del Departamento

\footnotetext{
Universidad de Tarapacá. Arica-Chile. E-mail: dquirozthompson@hotmail.com

Universidad de Tarapacá. Departamento de Ciencias Históricas y Geográficas. Arica-Chile. E-mail: albertodiaz@uta.cl

Universidad de Tarapacá. Departamento de Ciencias Históricas y Geográficas. Arica-Chile. E-mail: lgaldame@uta.cl

4 Universidad de Tarapacá. Departamento de Ciencias Históricas y Geográficas. Arica-Chile. E-mail: rruz@uta.cl
} 
de Arica". De acuerdo con las disposiciones de la Ley $\mathrm{N}^{\circ} 13.039$ que le dio origen, las funciones de la JAA eran "realizar las obras que se estimen necesarias para el adelanto de Arica, el fomento de la producción y de su comercio; y para el bienestar general de sus habitantes"3. Según su marco legal, el desarrollo y progreso del Departamento se convertían en un imperativo ineludible para las políticas locales propuestas. Siguiendo una perspectiva "desarrollista"4, se procuraría el progreso de todo el Departamento, buscando el mayor aprovechamiento de los recursos naturales de la zona cordillerana ${ }^{5}$; de esta manera, el papel de los productores andinos estaría dado por las actividades agropecuarias (ver Mapa 1). A partir de 1959 la JAA comenzó a formular los primeros planes de desarrollo de los sectores rurales del Departamento de Arica ${ }^{6}$, proyectando la elaboración de un diagnóstico de la biología terrestre y de los recursos naturales de la zona. Los resultados del estudio dieron origen a dos planes de desarrollo: el Plan auquénidos de 1960 y Plan de Desarrollo Agropecuario del año $1961^{7}$, siendo este último del cual nos ocuparemos en el siguiente apartado.

\section{Plan de Desarrollo Agropecuario del Departamento de Arica (1961-1965)}

Este programa fue resultado de un convenio establecido entre la JAA y el Ministerio de Agricultura, en coordinación directa con la Corporación de Fomento (CORFO), la Dirección de Riego y la Caja de Colonización Agrícola ${ }^{8}$. Dicho convenio permitió a los agentes del Ministerio de Agricultura ejecutar una serie de proyectos financiados por la $\mathrm{JAA}^{9}$.

Los diagnósticos previos también habían determinado que algunos de los factores que minimizaban el nivel de productividad agropecuaria de los campesinos andinos eran: a) aprovechamiento parcial, muy bajo del recurso agua; b) ineficiencia de la empresa agrícola, vale decir, mal uso de los factores de producción (agua, suelos, fertilizantes, mano de obra, etc.), y con ello altos costos; c) comercialización ineficiente y desaprovechamiento de mercados potenciales con ausentismo empresarial exageradamente alto; y d) competencia de la producción "hortalicera" de Tacna ${ }^{10}$. Como medidas estratégicas el Plan de Desarrollo Agropecuario estableció: "Mejorar el aprovechamiento de los recursos agrícolas, particularmente del agua de riego... Mejorar la eficiencia de la empresa agrícola... Mejorar la comercialización de los productos agrícolas... y Mejorar las condiciones de vida de la población rural" "11. Una de las formas que se adoptó para capitalizar y reorganizar la producción fue el otorgamiento de créditos agrícolas de mediano y largo plazo a distintos productores de los valles de Azapa y Lluta. Con ello se consiguió la extensión de áreas de cultivos, la redistribución parcelaria, habilitación y legalización de predios y creación de colonias agrícolas. En ellas participaron la Empresa de Comercio Agrícola, "E.C.A., para el poder comprador de rubros básicos, Endesa para la electrificación, Banco del Estado para los créditos operacionales a corto plazo, Fundación Viviendas de Emergencia para el plan habitacional campesino y Compañía Chilena de fertilizantes, stocks y precios guano blanco" 12 . El énfasis dado a los valles de Azapa y Lluta se habría debido a su mayor producción agrícola. De hecho, en la década de 1960 dichos valles abastecían a los mercados de Arica y del Norte Grande, principalmente con frutales, hortalizas y alfalfa y con primicias que eran comercializadas en el centro y sur del país ${ }^{13}$. Así, se incentivó la introducción de créditos dirigidos a mejorar el aprovechamiento del recurso hídrico y con ello aumentar los cultivos de los valles ${ }^{14}$. Los créditos para los campesinos andinos debían ser invertidos en sistemas de regadío eficientes, como lo constituía el caso del método por aspersión ${ }^{15}$, sistema moderno y con capacidad para duplicar el aprovechamiento de agua para el regadío ${ }^{16}$. Del mismo modo, como era indispensable racionalizar el uso y distribución de agua, la JAA colaboró en la construcción de la central hidroeléctrica de Chapiquiña ${ }^{17}$. Esta obra permitió asegurar en parte el suministro eléctrico en la ciudad de Arica, además de aumentar el agua de regadío y su distribución en canales por los diferentes sectores del valle de Azapa ${ }^{18}$. Mientras en Lluta el Plan generó la elaboración de estudios sobre la calidad química de las aguas del río y algunos ensayos de cultivos regados con diversas calidades de agua, aparte de obras de control sobre los ríos Colpitas y Azufre, tributarios del Lluta ${ }^{19}$. Para entonces, sólo se utilizaba el $16 \%$ del recurso agua, por lo que era necesario mejorar la canalización y distribución para aumentar la superficie cultivable del valle, la cual se estimaba que podía alcanzar hasta 3.300 hectáreas de cultivos semipermanentes (alfalfa principalmente) ${ }^{20}$. 


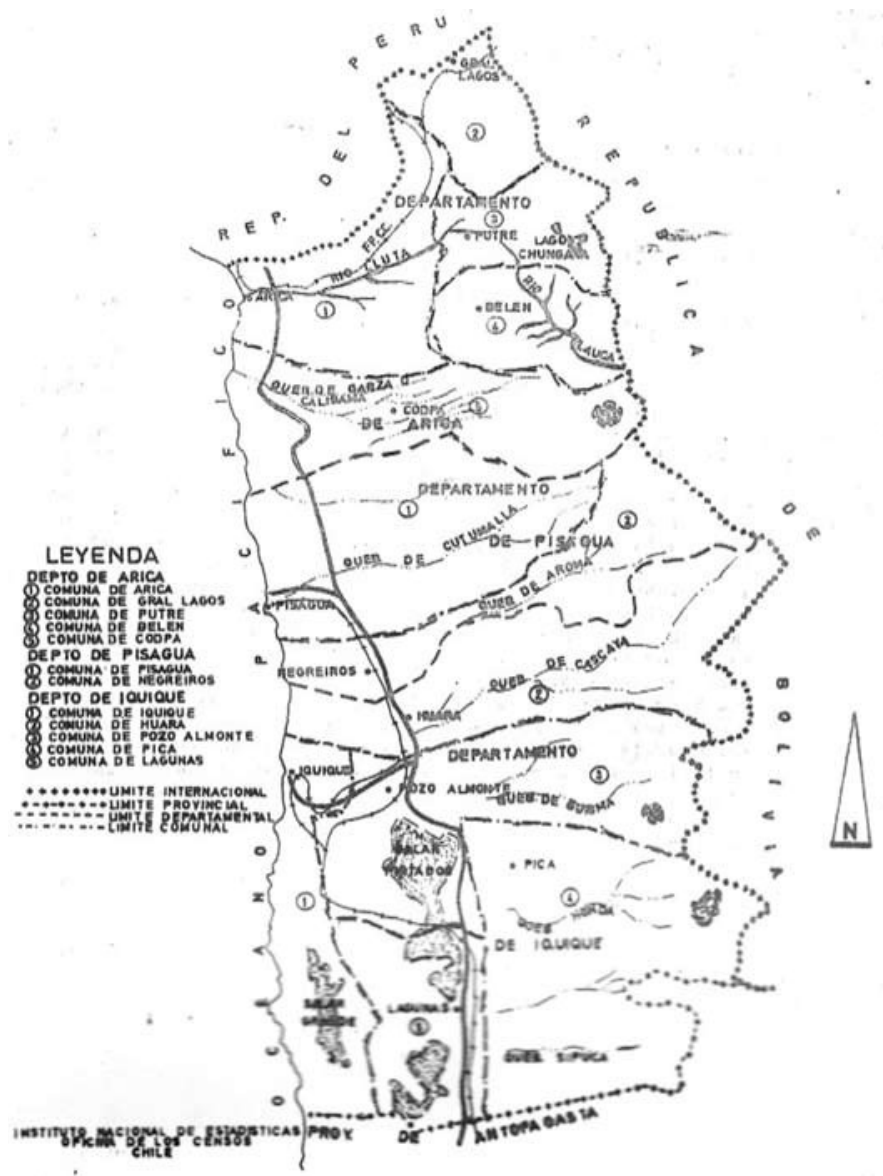

Mapa 1. Croquis del Departamento de Arica, 1970.

Fuente: Entidades de Población XIV Censo Nacional de Población y III de Vivienda 1970, p. VIII.

\section{Reformulación del Plan de Desarrollo Agropecuario (1967-1972)}

Para 1963, una comisión evaluadora del Plan de Desarrollo Agropecuario determinó que existían algunos problemas para fomentar las actividades productivas en la precordillera y altiplano de Arica, como lo eran la comercialización de los productos, debido a la lejanía de las localidades, a la inexistencia de rutas habilitadas y al alto costo de los fletes ${ }^{21}$. Sumemos a esto el inadecuado estado sanitario del ganado por la falta de conocimientos veterinarios y a la falta de agua de riego para mantener una adecuada extensión de pastales para la alimentación de la masa ganadera de camélidos y ovinos en la sierra.

Respecto de la producción agrícola, la comisión analizó los cultivos de las comunidades de la sierra de Huaylillas como Putre, Belén, Ticnamar y Taruguiri, clasificándolas en dos tipos. Uno, los "cultivos generales" compuestos por papa, alfalfa y maíz destinados al autoconsumo. Dos, los "cultivos especiales" correspondían al cultivo del orégano que era considerado un producto exportable. Los obstáculos para la producción del orégano estaban dados por la falta de agua (y no solo por su escasez) sino también por su distribución. Para este fin, la JAA se abocó al mejoramiento de canales, estanques y acumuladores. En tal sentido, para promover el cultivo del orégano el Plan de Desarrollo Agropecuario se ocupó de los canales de regadío en la precordillera, a través de un plan de manejo eficiente de los recursos de agua ${ }^{22}$.

En 1967 la JAA reformuló los objetivos del Plan Agropecuario vinculándose con el Plan Andino, el cual entregaría asistencia técnica para el 
funcionamiento de los nuevos proyectos de fomento productivo $^{23}$. La mayoría de los programas derivados de esta reformulación buscaron afrontar los problemas agrícolas que afectaban a la zona rural andina y que podríamos resumir en tres aspectos: i) tenencia de la tierra, ii) ineficiente aprovechamiento de los escasos recursos de agua para regadío y iii) manejo inadecuado del ganado.

\section{i) Tenencia de la tierra}

Según el Plan Agropecuario, la tenencia de la tierra era uno de los puntos más complejos de la zona interior del Departamento. Por ejemplo, el tipo de tenencia en el área andina estaba dado por el fraccionamiento de los terrenos (minifundio), irregularidad en la tenencia, posesión de grandes superficies por sucesiones, muchos años sin miembros residentes en el país, minimización de las rentas como consecuencia del "parvifundio", dificultad de la comercialización y el aislamiento, abandono de los predios por los agricultores y ganaderos y su entrega en mediería ${ }^{24}$. Este conjunto de aspectos serían la causa de la baja producción de la zona andina y del mayor fraccionamiento de la propiedad. En otro diagnóstico de la JAA, se identificaron dos tipos de tenencia de la tierra en la precordillera, relacionados uno con los terrenos de riego y el otro con los terrenos de secano ${ }^{25}$.

Ahora bien, los proyectos del Plan Agropecuario a partir de 1967 estuvieron dirigidos sólo a las comunidades fortalecidas en términos organizacionales, es decir, "las que están suficientemente organizadas y preparadas para un buen uso de los fondos, materializados en insumos para la construcción de las obras propuestas" 26 ; considerándose las comunidades precordilleranas de mayor productividad. La mayoría de las obras de infraestructura estuvieron dirigidas al mejoramiento del deficiente regadío, acentuado además por la aguda sequía registrada entre los años 1965-1972, lo que supuestamente al parecer agravó los problemas de la baja productividad agropecuaria y deficiente organización de los campesinos andinos de la precordillera y altiplano ariqueño. En este escenario, la JAA diseñó las siguientes estrategias:

1. Política de arraigo de los naturales de la zona, incluyendo el regreso de los que han emigrado, para los que se crearían planes productivos:

a) desarrollo de actividades económicas diferentes de las tradicionales tales como artesanía y crianza de animales de pieles, b) mejoramiento de la actividad agropecuaria tradicional,

c) solución de los actuales problemas de abastecimiento.

2. Expropiación de terrenos de propietarios ausentes o que están arrendando y terrenos mal aprovechados.

3. Aceleración del proceso de constitución legal del dominio de la propiedad individual y comunitaria.

4. Reorientación por parte del crédito destinado a insumos para la formación de unidades económicas $^{27}$.

Los programas propiciados por la JAA buscaron aumentar la producción agrícola de los indígenas, pero ahora con conocimientos más profundos de la zona, con el fin de reformular el sistema tradicional de subsistencia por uno de generación de divisas.

\section{ii) Escasos recursos de agua para regadío}

En 1969, el Servicio Agrícola Ganadero (SAG) se integró al Plan Agropecuario para ejecutar obras tales como la construcción de estanques de regadío y forestación (y reforestación) en la sierra. Para el primer caso, entre los años 1969-1975 se llevó a cabo un proyecto de construcción de estanques y canales en los pueblos de Socoroma, Chapiquiña, Belén, Lupica, Murmuntane, Cobija y Ticnamar, intentando racionalizar la utilización de los recursos hídricos y aumentar la producción agrícola ${ }^{28}$. Para el segundo caso, el proyecto de forestación y reforestación en la precordillera, se utilizaron áreas sin aprovechamiento agrícola con otras especies forestales de altura ${ }^{29}$.

\section{iii) Manejo inadecuado del ganado}

En cuanto al manejo del ganado, el SAG junto al Plan Agropecuario generaron el Proyecto de Desarrollo Ganadero. Este se llevó a cabo entre los años 1968-1975 en las comunidades de Putre, Socoroma, Chapiquiña, Belén y Ticnamar, cuya función fue establecer un manejo adecuado del ganado bovino y ovino de la precordillera. Para dicho efecto, la JAA aportó materiales y reproductores a la comunidad, la que contribuyó con la mano de obra, como puede constatarse en el Cuadro 1.

En el poblado de Putre se concentraron los principales proyectos. Como muestra, en 1967 se construyeron dos corrales comunales en pampa Lluscuma para aprovechar las 550 has de alfalfa 
Cuadro 1. Cálculo de cantidad de mercaderías entregadas a las comunidades de precordillera por el P.M.A en función de su disposición de mano obra gratuita (1967).

\begin{tabular}{|c|c|c|c|c|c|c|}
\hline Poblado & Obra & $\begin{array}{l}\text { Población } \\
\text { activa }\end{array}$ & $\begin{array}{l}\text { Carga } \\
\text { familiar }\end{array}$ & $\begin{array}{l}\text { Jornadas } \\
\text { (escudos) }\end{array}$ & $\begin{array}{l}\text { Periodos } \\
\text { (meses) }\end{array}$ & $\begin{array}{c}\text { Peso/alimento } \\
\text { (toneladas) }\end{array}$ \\
\hline Ticnamar & Canal & 56 & 235 & 4.770 & 6 & 9,8 \\
\hline Ticnamar & Corral comunal & 56 & 235 & 2.500 & 3 & 5,4 \\
\hline Belén & Canales & 61 & 284 & 5.490 & 6 & 10 \\
\hline Belén & Estanques & 61 & 284 & 4.800 & 4 & 8 \\
\hline Belén & Drenajes & 61 & 284 & 2.440 & 2 & 4 \\
\hline Putre & Corrales & 118 & 434 & 9.435 & 12 & 37 \\
\hline Chapiquiña & Canal & 75 & 283 & 9.000 & 8 & 16,3 \\
\hline Chapiquiña & Estanque & 75 & 283 & 3.375 & 3 & 6,1 \\
\hline Socoroma & Canal & 68 & 272 & 8.160 & 8 & 15,1 \\
\hline Socoroma & Mov. tierra & 68 & 272 & 3.060 & 3 & 5,7 \\
\hline \multicolumn{7}{|l|}{ Putre, Chapiquiña, } \\
\hline Ticnamar, Socoroma & Forestación & 148 & & 17.760 & 12 & 21,3 \\
\hline Total & & 526 & 1.508 & 70.870 & 12 & 138,007 \\
\hline
\end{tabular}

Fuente: AHVD. Fondo Estudios Técnicos de la JAA, Plan Desarrollo Agropecuario, 1967.

en producción y racionalizar el manejo de 5.500 ovejunos y 750 bovinos. La construcción de los corrales fue financiada con fondos de la JAA y la mano de obra por los comuneros andinos. Los beneficios directos de esta obra fueron el aumento de 12 horas en el talaje de los animales, el cruzamiento por la existencia de reproductores en el centro de monta y la erradicación definitiva de los corrales de la población, principal objetivo del programa ${ }^{30}$ (ver Fotografía 1). Por otra parte, la JAA firmó un convenio con la CORFO en 1969, para iniciar un programa de desarrollo agropecuario y crear un centro de experimentación con el objetivo de probar nuevos sistemas de manejo de ganado, fertilizantes, introducción de nuevas especies, dar asistencia técnica a los ganaderos de la zona, mejorar la comercialización de los productos y el control sanitario del ganado ${ }^{31}$.También se intentó reemplazar los modos de producción tradicionales en relación a la esquila y al control sanitario. Para ello CORFO, primeramente con Iquitex y después con el Servicio de Cooperación Técnica (SERCOTEC), se coordinaron en un Plan para el mejor uso de la materia prima, lo que contempló la compra directa de la lana a los ganaderos del altiplano, con lo que además fomentaban la concentración de los ganaderos en los caseríos, evitando así su dispersión hacia Bolivia como al puerto de Arica $^{32}$.

Sintetizando, los programas destinados al mejoramiento del regadío y manejo adecuado del ganado significaron los de mayor éxito entre las comunidades, reflejado en la gran cantidad de obras de regadío y en la amplia concurrencia de los indígenas andinos a los programas fitosanitarios. Años más tarde (1975) es posible advertir un mayor uso por los campesinos de algunos elementos técnicos sugeridos en los programas de la JAA, como fue la utilización de vacunas sanitarias para el ganado tanto en la precordillera como en el altiplano. La diversidad de programas, planes y obras de la JAA motivaron la creación de un organismo que integrara las distintas acciones que se llevaban a cabo en el área rural. De esta forma, en un seminario sobre "Estrategia de Desarrollo del Departamento de Arica" organizado por la Oficina de Planificación Nacional (ODEPLAN) y la JAA, se acordó organizar en 1972 el Comité de Desarrollo del Interior, para recabar información sobre la cultura y los valores de los pueblos andinos, intentando contextualizar las estrategias de Desarrollo de la JAA según las características socioeconómicas de la población indígena regional ${ }^{33}$.

\section{El Comité de Desarrollo del interior (1972-1976)}

En 1974, ya establecido el Comité y con la Junta Militar en el Gobierno, se desarrolló una nueva estrategia para el Departamento de Arica. No alejándose de los anteriores programas de 1961 y 1967 respectivamente, que buscaron el desarrollo agropecuario de la zona rural, el Comité generó un diagnóstico de la situación socioeconómica de los campesinos andinos de la precordillera y altiplano, 


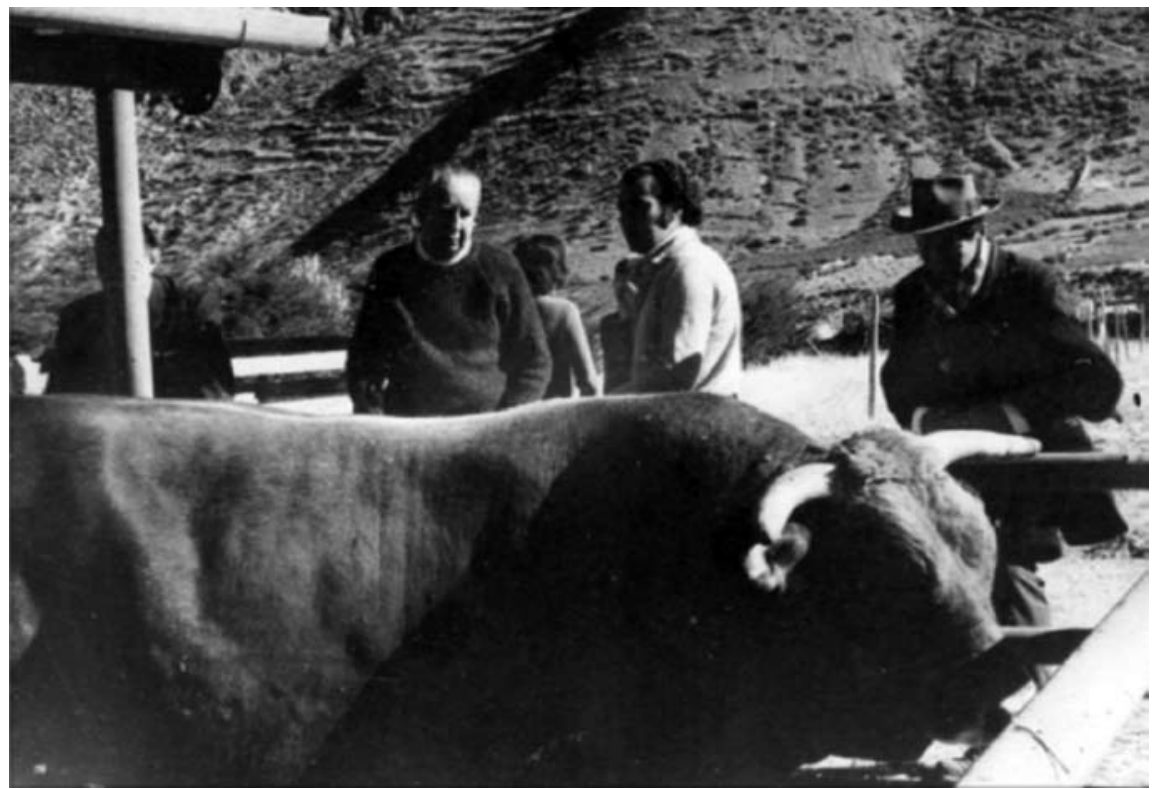

Fotografía 1. Políticas de fomento para la inserción de ganadería vovina en el Departamento de Arica (Estación de Monta de Putre a $3.550 \mathrm{msnm}$, aproximadamente década de 1960).

Fuente: Fondo Fotográfico Enrique Flores (AHVD).

información contenida en el documento "Esquema para una Programación del Desarrollo del interior del Departamento", en el cual se advierte que:

1. Necesidad de darle, a la comunidad, una estructura social (grupal) más definida, funcional y organizada.

2. En general, una evidente necesidad de elevar su actual estándar de vida.

3. Necesidad de una educación más de acuerdo con las exigencias del medio.

4. Necesidad de aumentar su productividad para integrarles a la economía regional

5. Necesidad de dotarles de los medios de comunicación adecuados para permitirles una integración progresiva al medio regional y nacional, tanto social como económicamente.

6. Necesidad de asegurarles una cierta regularidad en sus recursos de agua, fuente fundamental de su economía.

7. Necesidad de elevar su estándar tecnológico para aumentar su productividad y su estándar de vida.

8. Necesidad de dotarles de los medios adecuados de sanidad y mejoramiento de su dieta alimenticia.

9. Necesidad de descubrir nuevas fuentes de productividad.
10. Necesidad de darles asesoría concreta en materia de mecanización y abonos para incrementar rendimientos agrícolas.

11. Necesidad de asesoría en materia de mejoramiento de su ganadería y de la explotación ganadera.

12. Necesidad de contar con un cuadro sociodemográfico económico de los habitantes de la región.

13. Necesidad de contar con un cuadro claro de las condiciones de habitabilidad de la población.

14. Necesidad de proveerles suficientemente de modo que no sientan la necesidad imperiosa de emigrar.

15. Necesidad de facilitarles los medios que satisfagan sus necesidades de mercadeo y comercialización.

16. Necesidad de asegurarles un mínimo en materia de sana recreación.

17. A todo lo anteriormente enumerado, debemos agregar las necesidades básicas relacionadas con aspectos de interés nacional ${ }^{34}$.

De este modo, el informe integró algunos aspectos geopolíticos, subrayando que el despoblamiento de la cordillera debido a la migración a las ciudades de los campesinos e indígenas andinos se transformaba no sólo en un problema para el desarrollo del área 
rural, sino también para el resguardo fronterizo y la seguridad nacional. Por consiguiente, las acciones de las agencias gubernamentales vinculadas con la JAA procuraron poblar la cordillera (o hacer presencia efectiva en ella) con el establecimiento de servicios públicos y de organismos de seguridad y militares, aumentando la infraestructura vial y de servicios, en miras de los planes de regionalización. Otro aspecto destacado en el informe puso énfasis en la necesidad de poseer un mayor conocimiento de las condiciones socioculturales de las poblaciones andinas, tal como en 1967 se advirtió el interés por conocer la "idiosincrasia de las comunidades"35.

\section{Comuneros andinos en los sistemas crediticios agrarios}

Si consideramos la oferta económica que promovió la JAA a las organizaciones campesinas con créditos agrarios, planes de fomento ganadero y lechero como lo constituyeron el Plan de Desarrollo Agropecuario y Plan Lluta, entre otros, identificaremos que algunos campesinos andinos fueron regulares beneficiarios, quienes aprovecharon la coyuntura crediticia que ofreció la JAA para desarrollar la producción agrícola en toda el área rural. Algunos casos señalan dichas acciones, las cuales se describen a continuación:

\section{Agrupación Campesina Andina (ACA)}

La $\mathrm{ACA}^{36}$ estaba formada en su totalidad por población de origen étnico aymara, siendo una organización que accedió a un número importante de beneficios ${ }^{37}$. No cabe duda del conocimiento que los indígenas andinos poseían respecto de la administración de las entidades estatales, pues de ello dependía que sus solicitudes fueran aceptadas. Tampoco es menor el esfuerzo que habían realizado para poner en funcionamiento los suelos para la agricultura en los altos de Azapa. En su accionar, la ACA poseía una carpeta de proyectos, solicitudes y oficios requiriendo el apoyo de la JAA para financiar sus propuestas en el plano agropecuario ${ }^{38}$. Pese a que existían obras que debían realizar los propios campesinos, ya que la Corporación de Reforma Agraria (CORA) había entregado sitios en Azapa " $y$ todos los trabajos que allí hay que realizar son de cargo de las personas a quienes entregó el terreno", sin embargo la ACA solicitó la colaboración de la JAA. Años antes, los campesinos de esta agrupación habían experimentado con los programas de la JAA, dado que "los comuneros de algodonal son gentes provenientes de Surire, Guallatire, Socoroma, es decir, es gente del interior con quienes el Plan Andino ha estado trabajando durante mucho tiempo y siempre recurren a él cuando tienen dificultades"39. La recurrencia en las solicitudes que hacía la ACA quedó de manifiesto en la capacidad de articulación social y política de algunos de sus dirigentes, como fue el caso de Joselino Castro Fernández, asesor de algunas comunidades andinas ante las agencias gubernamentales ${ }^{40}$.

\section{Pueblo de Esquiña}

El caso de Dámaso Mamani, presidente de la Junta vecinal $N^{\circ} 35$ de Esquiña, es otro ejemplo de esta capacidad de promover y gestionar beneficios al interior de su comunidad. Este dirigente en 1973 dirige una carta a la JAA solicitando "1. Habilitación de terrenos para cultivo agrícola con un bull-dozer; 2. Ensanche acequia de regadio sector importante del valle por la misma máquina; 3. Construcción estanque de capacidad de mil doscientos metros cúbicos (Quiguetama); 4. Terminación estanque de regadío de los huertos del pueblo de Esquiña; 5. Construcción escuela y policlínico; 6. Construcción camino parte baja del valle de Esquiña y que continúe hacia el valle de Camarones; 7. Terminación camino Esquiña-Sahuara y caseríos que se mencionan"41. Para que su solicitud fuese aceptada, Mamani asiste a la sesión de Consejo de la JAA y expone allí las razones y la necesidad de estas obras, argumentando que "los terrenos del valle de Esquiña son de gran productividad, pero debido a la gran pendiente de ellos realmente no pueden producir lo que el valle produce. Por esta razón, (...) han solicitado un bull-dozer para habilitar el terreno y dejarlo en mejores condiciones de producción, sobre todo para la plantación de alfalfa que es el fuerte de la producción del valle. Señala seguidamente que si no hay algún organismo estatal que les pueda prestar esta ayuda, que les conceda un crédito, el cual pagarían" 42 .

Recapitulando, los casos descritos reflejan en parte el modo en que los productores andinos adoptaron las ofertas comerciales que brindaba la JAA, gestionando ante las entidades pertinentes y buscando soluciones para afrontar las dificultades que el mercado regional presentaba. No obstante, la programación local del desarrollo 
rural, la generación de proyectos, el apoyo financiero y la articulación entre la JAA con las comunidades campesinas andinas, relaciones incluso sociales construidas por largos años, se vieron truncadas por el D.L 1.612 de 1976, el cual estableció que "la Ley de Regionalización ha asignado las tareas para el desarrollo económico y social a los Intendentes Regionales apoyados por las Secretarías Regionales de Planificación y Coordinación y el Fondo Nacional para el Desarrollo Regional; (...) que lo anterior hace necesario readecuar la Junta de Adelanto de Arica al nuevo proceso dispuesto en forma coherente con la administración general del país (...) [de esta manera] Créase la Secretaría Ejecutiva de la Gobernación Provincial de Arica [SEGA] con personalidad jurídica y patrimonio propio, que se regirá por las normas del presente decreto ley y del Reglamento que se dicte" ${ }^{4}$.

\section{Comentarios}

Vistos los antecedentes sociohistóricos, es necesario consignar la articulación de las políticas agrarias a nivel local por parte de los productores agropecuarios andinos del Departamento de Arica durante la segunda mitad del siglo XX. Tal como advertimos, fue un periodo en que la institución local denominada Junta de Adelanto de Arica estimuló, bajo una perspectiva del progreso económico, el desarrollo del campesinado regional. En tal escenario, algunos productores andinos participaron activamente de los programas y proyectos implementados por la JAA, y, dicho sea de paso, este proceso de políticas desarrollistas en el ámbito agropecuario significó un aprendizaje para los indígenas respecto de cómo funcionaba el mercado capitalista, aportando a un número no menor de productores agrícolas de conocimientos que en décadas posteriores facilitarían su inserción en los mercados urbanos, ferias y en los terminales agropecuarios del Norte Grande de Chile.

En síntesis, los mayores recursos económicos del Departamento de Arica derivados del adelanto industrial permitieron la construcción de redes viales, infraestructura, viviendas, oportunidad laboral y mayores expectativas de desarrollo urbano. Cuánto de esto impactó efectivamente a los campesinos andinos es una difícil pregunta, pero que, conjeturamos, representaría un camino para acercarse a comprender el posicionamiento actual de un contingente de población indígena que tiene influencia en los mercados regionales entre el campo y la ciudad ${ }^{44}$.

\section{Literatura Citada}

Archivo Histórico Vicente Dagnino (AHVD). Fondo Estudios Técnicos de la JAA.

Dauelsberg, P. et al.

1972 Enciclopedia de Arica. Editorial de Enciclopedias Regionales Ltda., Santiago-Chile.

De Gregori, C. y L. Huber.

2005 Cultura, Poder y Desarrollo rural, Seminario permanente de investigación agraria. Perú.

Díaz, A.

2004 De la cordillera al mar. Una reflexión histórica sobre los Aymaras Chilenos y los mercados regionales. Boletín IFP, Año 2, $\mathrm{N}^{\circ} 8$.

Espina, L.

1971 Estudio Agroeconómico del valle de Azapa. Editorial Universitaria, S. A., Santiago-Chile, 50.
Galdames, L. y R. Ruz

2010 La Junta de Adelanto de Arica y John V. Murra. Dos lecturas sobre el desarrollo andino en el norte de Chile. Chungara, Revista de Antropología, volumen 42, $\mathrm{N}^{\circ} 1$.

\section{González, H.}

1998 La posición de Aymaras chilenos y bolivianos en la estructura de tenencia de la tierra de los valles de Lluta y Azapa. Taller de Estudios Andinos (TEA) Serie Documentos de Trabajo, Arica-Chile.

Ministerio de Hacienda

1958 Ley 13.039 crea la Junta de Adelanto de Arica. En http://www.leychile.cl/Navegar?idNorma=27344\&buscar $=$ ley+13.039 


\section{Notas}

1 John Murra en la década de 1970 propuso el modelo de economía vertical de aprovechamiento productivo, según el cual las poblaciones cordilleranas acceden complementariamente al control y manejo de diversos pisos ecológicos (valles bajos, precordillera y altiplano); adaptándose a las condiciones geográficas andinas, es posible extrapolar el modelo para explicar también la capacidad adaptativa de las poblaciones andinas, no solo a condiciones ecológicas determinadas sino también a escenarios sociopolíticos y étnicos diferenciados. Siguiendo este modelo, las disciplinas como la etnohistoria, antropología e historia regional han dado cuenta de la capacidad de ajuste y acomodo de las comunidades andinas a contextos coloniales y republicanos, por medio de la generación de estrategias indígenas y/o campesinas que les han permitido interactuar con elementos propios de la modernización, tales como relaciones comerciales mercantiles, creciente urbanización de las áreas costeras, extensión del control burocrático e instauración de relaciones económicas de tipo capitalistas.

2 Esta activación es producto de lo que ha sido denominado como "Puerto Libre" y corresponde al periodo de supresión de las franquicias aduaneras del puerto de Arica a partir del año 1953. Basado en un decreto supremo, esta liberación de gravámenes trajo consigo la llegada de una alta cantidad de comerciantes y mercaderías exóticas a la ciudad, la apertura de casas comerciales e incipientes industrias trayendo consigo un crecimiento explosivo de la ciudad de Arica, contabilizado en una constante de 400 personas diarias que buscaban trabajo, población proveniente principalmente de las cerradas oficinas salitreras. Complementada con la creación de la Junta de Adelanto de Arica en 1958, el puerto libre vio su supresión definitiva en 1962 con una nueva ley sobre nuevas normas para la exportación e importación del país; las iniciativas y objetivos de esta política comercial local fueron eliminados, creándose un nuevo marco jurídico para el desarrollo del Departamento de Arica en el extremo norte de Chile.

3 Ministerio de Hacienda. 1958. Ley 13.039 crea la Junta de Adelanto de Arica. Art. 2

4 Por "desarrollismo" entendemos al modelo conceptual clásico instalado en Latinoamérica entre la década de 1950 y 60 , y que se basó en la convicción de que la pobreza y el subdesarrollo podrían resolverse con inversiones adecuadas en capital físico e infraestructura. La teoría de la modernización percibía el desarrollo fundamentalmente como crecimiento económico, implicando así un mejoramiento de las condiciones de vida de sociedades tradicionales, diseñadas bajo criterios capitalistas de racionalización productiva y mayor aumento de sus capacidades de ingreso. Cfr. De Gregori y Huber. 2005. Cultura, Poder y Desarrollo rural, Seminario permanente de investigación agraria. Perú.

5 Uno de los objetivos de la JAA promovía "la prospección general de todas las riquezas naturales, terrestres o marítimas de la zona; y la planificación sobre la utilización de los recursos ubicados". Ministerio de Hacienda. 1958. Ley 13.039 crea la Junta de Adelanto de Arica. Art. 2.

6 De esta manera, "en conformidad al acuerdo 120, de fecha 14 de noviembre de 1959, se efectuarán diversos estudios en el interior del Departamento de Arica, tendientes a programar una serie de trabajos destinados a incrementar el progreso agrícola y ganadero de dicha zona, aumentando su capacidad de agua y fomentando la crianza de auquénidos en la precordillera", Archivo Histórico Vicente Dagnino (en adelante AHVD). Fondo Estudios Técnicos de la JAA, Vol. 172, 1960.

7 AHVD. Fondo Estudios Técnicos de la JAA, Índice convenios JAA/Ministerio de Agricultura, 1961-1967.

8 De esta forma, "según el acuerdo $N^{\circ} 551$, Plan de Desarrollo Agropecuario, de fecha 19 de abril de 1961, se faculta un convenio entre la Junta de Adelanto de Arica (JAA) y el Ministerio de Agricultura (...) la junta, interesada en el progreso de la agricultura ha destinado fondos para desarrollar sus posibilidades, conforme a lo expuesto en un plan agrícola (...) requiriendo especialistas y profesionales que la junta carece, en cambio el ministerio dispone (...) por lo tanto, con el objeto de que el ministerio cumpla de mejores formas sus funciones se suscribe este convenio". AHVD. Fondo Estudios Técnicos de la JAA, Acta de Resolución, 1961.

9 Las que correspondían a áreas de interés, tales como: Recursos naturales compuesta por los proyectos: $i$ ) reconocimiento de suelo y ii) estudio calidad química de las aguas del río Lluta; área Estudios Económicos: i) mercados, ii) mercadeo y iii) administración rural (FAO); área Campo Experimental de Azapa: i) introducción de nuevos cultivos y variedades mejoradas, ii) producción de frutales, forestales, semillas, etc., para venta precios de fomento, facilitando la diversificación; área Asistencia Técnica: i) extensión agrícola y ii) siembras demostrativas; área Fomento Ganadero: i) estación monta en Putre, ii) campañas ganaderas sanitarias y iii) entrega de reproductores; área Conservación de Recursos: i) obras manejo de riego, ii) nivelación de terrenos y iii) recargas napas subterráneas; área Control de Plagas: i) creación brigada de equipos de control de plaga; y área Lluta y Azapa: i) proyecto lechero Lluta, ii) proyecto hortalicería Azapa, iii) proyecto fruticultura Azapa, iv) proyecto cooperativa Juan Noé y v) proyecto habitacional campesino. AHVD. Fondo Estudios Técnicos de la JAA, Informe Esquema de Planificación del Desarrollo Agropecuario de Arica, 1962.

10 AHVD. Fondo Estudios Técnicos de la JAA, Informe Esquema de Planificación del Desarrollo Agropecuario de Arica, 1962.

11 El mejoramiento del ganado del altiplano no se consideró en este convenio, debido a que esta labor fue encomendada en 1960 al "Profesor de producción pecuaria de la Universidad de Chile Dr. Adolfo Albornoz Bustamante para que hiciera un completo estudio del clima, flora, fauna y suelo del altiplano Ariqueño" (50). De este estudio se concluyó que la población de auquénidos constituía "un rubro legítimo y natural de producción ganadera del Departamento de Arica; y su número y calidad deben ser aumentados y mejorados a través de un proyecto ganadero: plan auquénidos". Para llevar a cabo este plan era indispensable la organización de una Estación Experimental de Auquénidos y Animales de Pieles Finas, que fuera dependiente de la Junta de Adelanto de Arica y la Universidad de Chile (51). Dicha Estación fue construida en un sector del altiplano llamado Misitune 
y allí operó desde 1960 a 1965, fundamentalmente con el objetivo de aumentar la masa ganadera, calidad de lana, carne y cuero del altiplano. AHVD. Fondo Estudios Técnicos de la JAA, Informe Esquema de Planificación del Desarrollo Agropecuario de Arica, 1962.

12 AHVD. Fondo Estudios Técnicos de la JAA, Vol. 391, Informe Esquema de Planificación del Desarrollo Agropecuario de Arica, 1962, 2, 3.

13 Leónidas Espinas Estudio Agroeconómico del valle de Azapa, JAA-Universidad de Chile, Facultad de Agronomía, Departamento de Ciencias Económicas y Sociales, SantiagoChile, 1971, 50.

14 De esta forma se dispuso que "con el objeto de superar la baja eficiencia expuesta la CORFO concederá créditos controlados [...] exigiéndose la adopción de prácticas culturales no tradicionales”. AHVD. Fondo Estudios Técnicos de la JAA, Vol. 391, Esquema de Planificación del Desarrollo Agropecuario de Arica, 1962.

15 Consistía en conducir el agua por medio de cañerías colocadas sobre la superficie del suelo, con la ayuda de unos aspersores y con la presión que se ejerce sobre el agua, esta se eleva y cae pulverizada simulando una lluvia.

16 Se buscaba reemplazar los sistemas de regadío tradicionales del valle, entre los cuales podemos nombrar: 1) El sistema de "caracol" que es un método de riego superficial compuesto de surcos que se hacen en la tierra en forma de zigzag que pueden alcanzar una longitud de alrededor de 80 a 100 metros. Cada 25 metros se introducen en los surcos unas hendiduras por donde ingresa el agua de riego. La forma serpenteante de los surcos aminora la velocidad del agua, con lo cual se adapta a las altas pendientes de algunos sectores del valle, disminuyendo la erosión y la infiltración del agua. Sin embargo, absorbe una gran proporción de mano de obra y tiene un elevado costo de producción en la extracción de aguas subterráneas como producto de la infiltración de las aguas superficiales en el relleno aluvial. 2) El sistema superficial por inundación que se utiliza en el cultivo de olivos es un sistema por tasas que consiste en un pretil circular o cuadrangular que rodea el árbol. Espina, Leónidas óp. cit., 50.

17 Por medio de un convenio con la Empresa Nacional de Electricidad S.A. (ENDESA), cediendo $10 \%$ de su presupuesto para financiar el $80 \%$ del gasto total de la obra. A su vez, ENDESA entregó a la JAA “acciones de su empresa por el valor equivalente a los aportes de la junta (...) estas acciones serán reemplazadas por acciones de una sociedad que ENDESA y la JAA deberán formar, a fin de obtener el máximo de economía en el costo de producción de energía eléctrica una vez terminada la planta". La central de la que hablamos se ubica a $80 \mathrm{~km}$. al oriente de Arica, en la ladera sur de una quebrada llamada Chusmiza y que es un afluente del río Livilcar y quebrada de Azapa, enclavada en la ladera de tal forma que aprovechaba el trayecto del canal Lauca. AHVD, Actas de Consejo de la JAA No 105, sesión 2 de agosto 1960.

18 Dichos canales seguían el curso de una matriz central que formaba parte de la infraestructura del canal Lauca, el cual nacía en una bocatoma situada a $60 \mathrm{~km}$ al interior de Arica y a $950 \mathrm{msnm}$. Aprovechando el canal matriz se construyó un sistema de canales derivados y subderivados que suministraron agua a diferentes sectores agrícolas, tales como los "canales derivados" de Surire, La Cruz, 18 de Septiembre, Sobraya sur, Sobraya norte, Cerro Blanco, Cabuza, Chuval, Cerro Moreno, San Miguel, Las Riveras, Alto Ramírez, Alto Ramírez norte, Pago de Gómez sur, Pago de Gómez norte; y "canales subderivados" en Alto Ramírez, Pago de Gómez sur, Ordóñez, SENDOS, Campo Verde y desagüe. Obras Hidráulicas, Vol. 6331 ENDESA, 1961, Archivo Nacional Siglo XX, Santiago-Chile, citado en Contreras y Luna, óp. cit., 122, 123.

19 AHVD. Fondo Estudios Técnicos de la JAA, Vol. 391, Esquema de planificación del Desarrollo Agropecuario de Arica, 1962, 4,5.

20 En el año de 1960 contaba con 5.000 hectáreas de las cuales 2.200 correspondían a superficie regable, de ellas 230 estaban cultivadas con praderas de alfalfa, 1,14 con maíz y 40 con hortalizas varias.

21 AHVD. Fondo Estudios Técnicos de la JAA, Plan de Desarrollo Agropecuario, 1967.

22 AHVD. Fondo Estudios Técnicos de la JAA, Plan de Desarrollo Agropecuario, 1967,16.

23 Consignemos que el Plan Andino fue un programa de desarrollo comunitario destinado a colaborar con el progreso de la población indígena, pertenecía a la OIT y se implementó en Arica por medio de la gestión de la JAA desde 1962 hasta aproximadamente 1976 .

24 La mediería corresponde a un convenio de explotación en la que el propietario de un predio entrega a un tercero la posibilidad de explotar sus terrenos a cambio del pago de una renta que puede corresponder a un porcentaje del total de la cosecha conseguida por el mediero. En general se trata de un convenio informal, de palabra. Existen otras modalidades de explotación de terceros como el arriendo que implica un contrato formal y escrito, y la custodia que se trata de un acuerdo verbal que permite a un tercero explotar un predio a cambio de mantenerlo en estado productivo. González (1998) sostiene que la mediería habría sido introducida masivamente en los valles de Lluta y Azapa por los campesinos andinos durante el periodo de asentamiento y habilitación de nuevos espacios agrícolas favorecido por CORA. En La posición de Aymara Chilenos y Bolivianos en la estructura de tenencia de la tierra de los valles de Lluta y Azapa. Taller de Estudios Andinos (TEA). Serie Documentos de Trabajo, Arica-Chile.

25 En los terrenos de regadío predomina "el tipo de propiedad individual, y en general se advierte un excesivo fraccionamiento, se constata la presencia de una fuerte proporción de minifundio, en su más simple definición como tenedores de una superficie inferior a la unidad agrícola familiar. Los sistemas de tenencia de estos loteos, en orden o relación a su cuantía son: masa tenedora de suelos fiscales, a las cuales se les tramita títulos gratuitos de dominio; propietarios; arrendatarios; y masa tenedora de terrenos particulares". La tenencia de tierras fiscales poseía problemas en cuanto a irregularidades en títulos gratuitos de dominio, mientras que los que detentaban tierras particulares, generaban problemas relacionados con el arrendamiento o la mediería a personas extranjeras, particularmente de origen boliviano. Mientras en los terrenos de secano, "el sistema de tenencia es mayoritariamente una mera ocupación de tierras fiscales y en menor grado, comunidades propietarias con títulos no saneados. Todos ellos tienen la posibilidad, 
y así lo están haciendo las comunidades de Ticnamar y Putre, de constituirse conforme a la legislación especial y vigente". AHVD. Fondo Estudios Técnicos de la JAA, Plan de Desarrollo Agropecuario, 1967, 4.

26 AHVD. Fondo Estudios Técnicos de la JAA, Vol. 152, 1967.

27 AHVD. Fondo Estudios Técnicos de la JAA, Vol. 152,1967.

28 Precisamente, los proyectos fueron revestimiento canal Aroma en la localidad de Socoroma; construcción canal pampa Lluscuma, Vilcabani en Putre; construcción estanque Misaña, estanque reguladores en Pukara, Chapaca, Calapampa y canal Aquechoco, revestimiento canales Aquechoco, Quispaquipane y Molino, todos en la localidad de Belén; construcción estanque de Chapiquiña; construcción estanque, canal principal de distribución del estanque anterior y Drenaje para regadío y captación de napas freáticas en Oxayupuya de la localidad de Tignamar; construcción de estanque de regulación de $1.500 \mathrm{~m}^{3}$ en Murmuntane, Lupica y Cobija; y construcción de tres estanques en Timar. AHVD. Fondo Estudios Técnicos de la JAA, Plan Desarrollo Agropecuario, 1967.

29 Las obras consistieron en la plantación de 112.500 plantas en pampa Lluscuma; 57.500 en Ticnamar, Oxa y Lupica y 6 mil en Chapiquiña. Complementariamente se realizó el sembradío de semillas y principalmente frutales, las que tuvieron mayor éxito en Socoroma, Belén, Ticnamar y Lupica, insertándose nuevas especies como almendros, manzanos, perales, duraznos y tunales. AHVD. Fondo Estudios Técnicos de la JAA, Vol. 339, 1969.

30 AHVD. Fondo Estudios Técnicos de la JAA, Vol. 146, 1975.

31 La JAA intentó proporcionar un mercado económicamente más rentable para los ganaderos, reemplazando sus antiguos circuitos comerciales tradicionales en donde "la lana producida en el altiplano era comprada por comerciantes bolivianos y chilenos y cancelada en dinero o en mercadería. Teniendo como principal inconveniente el pago en precio no real o su trueque en mercadería. AHVD. Fondo Estudios Técnicos de la JAA, Vol. 146, 1972.

32 En cuanto al manejo del ganado, se construyó una estación experimental de CORFO en Caquena. Ahí se enseñaban nuevas técnicas a los pobladores del altiplano, siendo la esquila mecanizada y el tratamiento enterotoxémico los programas más promovidos; estos últimos tuvieron gran aceptación, "extendiéndose en el primer y segundo periodo a Caquena y Chañupalca, en el tercer período a Parinacota y Chucuyo realizándose demostraciones también en Visviri. AHVD. Fondo Estudios Técnicos de la JAA, Vol. 146, 1972.

33 El giro al menos en teoría más cultural de este comité se habría debido en parte a la acción de profesionales y académicos, en mayor medida antropólogos de la Universidad de Chile. Hay que tener en consideración la influencia que en ese tiempo estaba teniendo para las zonas andinas, incluida Arica, el Modelo de John Murra, quien de hecho visitó la zona. Ver Galdames y Ruz. 2010. La Junta de Adelanto de Arica y John V. Murra. Dos lecturas sobre el desarrollo andino en el norte de Chile. Chungara, Revista de Antropología, volumen 42, No 1, 257-270.

34 AHVD. Fondo Estudios Técnicos de la JAA, Vol. 2, 1974.
35 Lo anterior es corroborado en un informe del Servicio de Planificación Comunal (SERPLAC) referente a la existencia de minifundios: "esta modalidad de cultivo ha generado minifundios cuya existencia se justifica por la poca superficie cultivable; la escasez de agua de riego en los meses de sequía: la diversidad de calidad de tierras en distintas partes, y la dificultad de canalización y riesgos de pendientes. Por las razones mencionadas, un mismo agricultor posee varios retazos en un mismo o distinto valles precordilleranos; ya que lo que produce en una hijuela, no lo puede cultivar en la otra, por lo tanto, esta multiplicación de minifundistas que se observa en los valles precordilleranos, obedece a características propias de la zona y la aplicación de una política de creación de unidades agrícolas familiares - que en terrenos planos en aconsejable- no aparece como muy apropiada para estos valles tan disímiles de aquellos del resto del país". AHVD. Fondo Estudios Técnicos de la JAA, Vol. 180, 1977.

36 El 17 de junio de 1974 se creó un acta de Asentamiento y el 30 de julio del mismo año un contrato con la CORA para establecer una Sociedad Agrícola de Reforma Agraria en los predios de Pampa Algodonal, Pampa Algodonal norte uno, Pampa Algodonal norte dos y Pampa Pan de Azúcar ubicados en los altos de Azapa, conformándose la Agrupación Campesina Andina.

37 En 1976 la "Agrupación Campesina Andina", por medio de Juan Marcos Chambe presidente del sector Surire y Joselino Castro Fernández presidente de ACA, envió un oficio a la JAA en donde expresaba: "1. La Sociedad Agrícola de Reforma Agraria "Agrupación Campesina Andina”, se encuentra efectuando trabajos de habilitación de suelos desérticos en terrenos pertenecientes a la Corporación de Reforma Agraria, en el Km 45 del Valle Azapa. 2. En uno de los sectores de nuestra SARA, denominado Surire, ubicado en una terraza de orientación sur respecto al río San José, hemos tenido varios problemas por fallas de conducción de agua para regadio. Los terrenos mencionados, están siendo cultivados gracias a que construimos un canal de 4.000 m.l. el cual nos ha permitido habilitar una superficie de 40 hás., de suelos vírgenes. Dichos suelos están siendo explotados con zanahorias, cebollas y otras hortalizas. 3. En la actualidad, existe el grave riesgo de perder gran parte de nuestros esfuerzos productivos, por el problema de conducción de agua. En efecto la primera etapa del canal mencionado fue construido en una escarpada ladera, de material no estratificado tipo ripio, encontrándonos luego de avanzar $500 \mathrm{mts}$. con una gran roca, que impidió continuar la obra. Dicho inconveniente fue solucionado mediante la construcción de una canoa, elevada bordeando la roca, de 14 metros de altura, sobre el lecho del rio San José y de 49 metros. La estructura mencionada, por los materiales usados, en repetidas ocasiones se ha desplomado, ocasionando la suspensión del riego en perjuicio de los cultivos, por los días de demora en su ejecución. 4. Nuestras metas de habilitación de suelos, proceso en el cual tenemos asesoría técnica de la CORA como asimismo el comodato de un Bulldozer, no se podrán cumplir mientras no solucionemos en definitiva el problema expuesto. En otras palabras, la estructura solo soporta escasamente el agua para regar los terrenos actualmente cultivados y con el riego descrito. 5. Ponemos en su conocimiento que 
concurrimos al Departamento de Desarrollo comunal de esa Junta de Adelanto, en el cual nos entrevistamos con el Sr. Carlos Solari para solicitarle el concurso del funcionario de esa Corporación Sr. Florencio Bustillos, especialista en trabajos de excavación y remoción de material mediante explosivos. 6. Con el citado funcionario efectuamos una visita a terreno, confirmando la posibilidad técnica de efectuar el trabajo, mediante la perforación y excavación de la roca y el paso por ella del canal de conducción de agua de riego, eliminando la totalidad de la estructura defectuosa descrita anteriormente. 7. Para desarrollar dicho trabajo el Sr. Bustillos mencionó la necesidad de contar con los siguientes elementos: compresora, brocas, 4 sacos de nitrato de amonio, 4 cajones de amón gelatina, una caja de guía, detonadores. 8. Considerando nuestra situación actual, en el sentido de que todos nuestros recursos económicos han sido destinados a los trabajos de habilitación, es que nos permitimos solicitar al Sr. Presidente su atención a nuestro problema considerando los fines que se persiguen. 9. Finalmente, tenemos a bien poner en su conocimiento que desde ya ponemos a disposición de las obras a realizarse, la totalidad de mano de obra necesaria, como asimismo el transporte y alimentación y alojamiento del personal especializado en la ejecución de la obra", AHVD. Fondo Estudios Técnicos de la JAA, Acta 1.079 "Solicita Cooperación para solucionar problema comunitario", 9 de agosto 1976.

38 Señalándose por ejemplo, “esto es un esfuerzo titánico que hace esa Agrupación en la parte interior del valle. Ha estado presente en los trabajos que han realizado y el obstáculo -tal como lo dicen en la carta-es este pasaje como de unos 15 a 20 metros. Considera que es de justicia hacer esta inversión ya que redunda en bien de Arica (...) y estima que es justo ir en ayuda de esta gente que ha puesto todo su esfuerzo en este trabajo". AHVD. Fondo Estudios Técnicos de la JAA, Acta 1.079 "Solicita Cooperación para solucionar problema comunitario", 9 de agosto 1976, 2,3.

39 AHVD. Fondo Estudios Técnicos de la JAA, Acta 1.079 "Solicita Cooperación para solucionar problema comunitario", 9 de agosto 1976, 2,3.

40 En 1974 este dirigente sugirió la necesidad de potenciar el conocimiento de la cultura andina dentro del sistema educacional que impartían las escuelas en el interior del Departamento, en esta ocasión se dirigió a la JAA: "Manifiesta el señor José Lino Castro que es del pueblo de Surire, en el limite con Bolivia, cerca esta el retén de Chilcaya y pertenece a la subdelegación de Codpa. Agrega que nació con la lengua aymará y toda su familia es aymara. Conoció el español a la edad de siete años cuando tuvo que asistir a la escuela de Camiña, en donde el profesor no lo entendía ni él al profesor. Muchos de los niños que en ese tiempo asistían a la escuela de Camiña, tenían el mismo problema, recibiendo malos tratos de parte del profesor por este hecho. Esto produjo que se sintieran humillados por no saber el idioma español. Señala seguidamente, que es muy importante que los profesores rurales que van a las escuelas del interior tengan un conocimiento del idioma Aymara, es importante que los funcionarios públicos tengan conocimiento de este idioma debido a que si no lo hacen la gente no los entiende y no se explican el motivo de su visita. También es importante para ellos que no se considere al idioma Aymara como propio de los bolivianos porque se sienten humillados, siendo chilenos. (...) El señor José Lino Castro manifiesta que a primera instancia, los funcionarios que llegan a los pueblos de la cordillera, en el límite con Bolivia, encuentran que el 50\% de la población no entiende español. Por esta razón, recalca los funcionarios que viajan a estos pueblos apartados deben tener ciertos conocimientos del Aymara, como primer contacto con el pueblo. Como segundo contacto, sería importante que los funcionarios escuchen primero la opinión del pueblo para hacer cualquier problema de desarrollo. Informa que el año pasado salió una disposición en todos los retenes de los pueblos del interior, en la reforestación del Lauca. Señala que los carabineros les prohíben comer la paja, les prohíben quemar la queñua y la yareta. Explica que esto para ellos es como quitarles sus herramientas de la mano, porque en primer lugar queman paja en los meses de julio y agosto para que en enero caygan las lluvias y rebrote el pasto nuevo el año siguiente, para los animales. Queman la yareta para espantar a los pumas. Indica que con esta medida los animales se van adelgazando porque no hay pastos", AHVD. Fondo Estudios Técnicos de la JAA, Vol. 15, Acta $N^{\circ}$ 11/74 Comité de Desarrollo del Interior, 2 de agosto 1974.

41 AHVD. Fondo Estudios Técnicos de la JAA, Comisión de Desarrollo del Interior Acta $N^{\circ} 15 / 74,6$ de septiembre de 1974, 6.

42 AHVD. Fondo Estudios Técnicos de la JAA, Comisión de Desarrollo del Interior Acta $N^{o}$ 15/74, 6 de septiembre de 1974, 6.

43 D.L.-1.612, 1976, disuelve la Junta de adelanto de Arica y crea la secretaría ejecutiva de la gobernación provincial de Arica, http://www.leychile.cl/Navegar?idNorma $=6688$ \&buscar=dl+1612, extraído el 15 de marzo de 2010.

44 Díaz, Alberto. 2004. De la cordillera al mar. Una reflexión histórica sobre los Aymaras Chilenos y los mercados regionales. Boletín IFP, Año 2, No 8, Estados Unidos. 